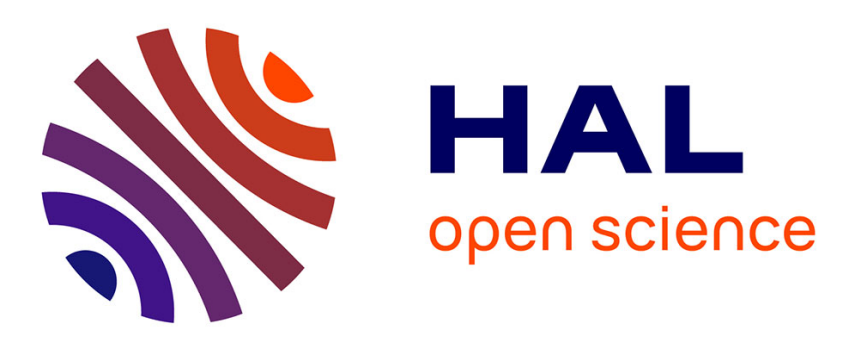

\title{
Visible and Ultraviolet Spectroscopy of Gas Phase Rhodamine 575 Cations
}

Steven Daly, Alexander Kulesza, Geoffrey Knight, Luke Macaleese, Rodolphe Antoine, Philippe Dugourd

\section{- To cite this version:}

Steven Daly, Alexander Kulesza, Geoffrey Knight, Luke Macaleese, Rodolphe Antoine, et al.. Visible and Ultraviolet Spectroscopy of Gas Phase Rhodamine 575 Cations. Journal of Physical Chemistry A, 2015, 119 (22), pp.5634-5641. 10.1021/acs.jpca.5b03187 . hal-01344597

\section{HAL Id: hal-01344597 \\ https://hal.science/hal-01344597}

Submitted on 12 Jul 2016

HAL is a multi-disciplinary open access archive for the deposit and dissemination of scientific research documents, whether they are published or not. The documents may come from teaching and research institutions in France or abroad, or from public or private research centers.
L'archive ouverte pluridisciplinaire HAL, est destinée au dépôt et à la diffusion de documents scientifiques de niveau recherche, publiés ou non, émanant des établissements d'enseignement et de recherche français ou étrangers, des laboratoires publics ou privés. 
Visible and Ultraviolet Spectroscopy of Gas Phase Rhodamine 575 Cations. S. Daly, A. Kulesza, G. Knight, L. MacAleese, R. Antoine and P. Dugourd. J. Phys. Chem. A 119, 5634-5641 (2015).

http://dx.doi.org/10.1021/acs.jpca.5b03187 


\section{Visible and Ultraviolet Spectroscopy of Gas Phase Rhodamine 575 Cations.}

Steven Daly ${ }^{\mathrm{a}, \mathrm{b}}$, Alexander Kulesza ${ }^{\mathrm{a}, \mathrm{b}}$, Geoffrey Knight ${ }^{\mathrm{a}, \mathrm{b}}$, Luke MacAleese ${ }^{\mathrm{a}, \mathrm{b}}$, Rodolphe Antoine $^{\mathrm{a}, \mathrm{b}}$, Philippe Dugourd ${ }^{\mathrm{a}, \mathrm{b} *}$.

${ }^{\text {a }}$ Université de Lyon, F-69622, Lyon, France.

${ }^{\mathrm{b}}$ CNRS et Université Lyon 1, UMR5306, Institut Lumière Matière.

KEYWORDS Rhodamine, gas phase, UV-visible action spectroscopy, TDDFT.

ABSTRACT: The visible and ultraviolet spectroscopy of gas phase rhodamine 575 cations has been studied experimentally by action-spectroscopy in a modified linear ion trap between 220 and $590 \mathrm{~nm}$ and by time-dependent density functional theory (TDDFT) calculations.

Three bands are observed that can be assigned to the electronic transitions $S_{0} \rightarrow S_{1}, S_{0} \rightarrow S_{3}$ and $\mathrm{S}_{0} \rightarrow\left(\mathrm{S}_{8}, \mathrm{~S}_{9}\right)$ according to the theoretical prediction. Whilst the agreement between theory and experiment is excellent for the $S_{3}$ and $S_{8} / S_{9}$ transitions, a large shift in the value of the calculated $S_{1}$ transition energy is observed. A theoretical analysis of thermochromism, potential vibronic effects and - qualitatively - electron correlation revealed it is mainly the latter that is responsible for the failure of TDDFT to accurately reproduce the $\mathrm{S}_{1}$ transition energy, and that a significant thermochromic shift is also present. Finally, we investigated the nature of the excited states by analyzing the excitations and discussed their different fragmentation behavior. We hypothesize that different contributions of local versus charge transfer excitations are responsible for 1-photon versus 2-photon fragmentation observed experimentally.

\section{Introduction.}

The photo-physics of organic chromophores such as the rhodamine family of dyes is of importance to a broad range of subject areas ranging from the behavior of dye lasers to the labelling of biomolecules for structural assignment, and as such there have been a great many studies of their solution phase properties. ${ }^{1-11}$ There has also been considerable effort in recent years devoted to the study of the properties of such chromophores in the solvent-free 
environment of the gas phase. ${ }^{12-18}$ The recent transposition of Förster Resonance Energy Transfer (FRET) to the gas phase has only increased interest in the gas phase properties of these dyes, as they have been used as both donor and acceptor chromophores in many of the systems studied thus far. ${ }^{19-24}$ In particular, the use of rhodamine 575 (R575) as the donor chromophore in several of these studies has made an understanding of its gas phase photophysical properties of high interest. Additionally, the study of the gas phase photo-physical properties of rhodamine dyes provides a benchmark for electronic structure calculations, which remain a challenging prospect.

R575 has been subject to several previous gas phase studies. Sassin et al. studied the dependence of the photo-fragmentation and fluorescence of R575 cations on the laser power and buffer gas pressure using a continuous Ar ion laser at 515 and $488 \mathrm{~nm} .^{25,26}$ They observed that the photo-fragmentation and fluorescence were dependent on both the laser fluence and gas pressure, which was interpreted as absorption of multiple photons following many collisional deactivation cycles. Forbes et al. measured the fluorescence excitation and emission of R575 cations, giving maximum values of $495 \mathrm{~nm}$ and $506 \mathrm{~nm}$ respectively. ${ }^{27}$ They also measured a quadratic dependence of the photo-fragmentation yield as a function of the laser power, and invoked a two photon mechanism for fragmentation following either internal conversion or intersystem crossing to explain this observation. Chingin et al. looked at the influence of the position of the carboxyl moiety of the carboxyphenyl group of R575 in solution and the gas phase, the influence of metal cation adducts on the position of the photofragmentation maximum, and the influence of the charge state. ${ }^{28,29}$ They showed that both the R575 cation and anion absorb at $488 \mathrm{~nm}$, but that the neutral form is optically dark, and that there is a significant blue-shift of the absorption maximum as the size of the metal adduct is increased. Greisch et al. found the dispersed fluorescence maximum of $497 \mathrm{~nm}$ following excitation at $458 \mathrm{~nm}$ and measured a triplet state lifetime of $3250 \mathrm{~ms}$ for R575 cations. ${ }^{30}$ 
Finally, Nagy et al. determined the fluorescence lifetime of R575 cations to be $5.65 \mathrm{~ns}$ following absorption of $477 \mathrm{~nm}$ photons. ${ }^{31}$

There are two common themes in each of these studies. Firstly, the absorption, emission and fragmentation properties have only been studied in the visible portion of the spectrum. Given that multi-photon processes are invoked in explaining the photo-fragmentation of R575, examination of the photo-physical properties of the higher lying singlet states is of interest. Secondly, each of the studies mentioned above have used either continuous or else high repetition rate lasers, and as such have studied the photo-physical behavior over irradiation times of the order of milliseconds to seconds. The use of low repetition rate lasers allows the photo-physical properties to be studied over a much shorter time period; in this case that of a single laser pulse of the order of a few nanoseconds. This would in effect eliminate the influence of the gas pressure on the excited state dynamics since the time scale for gas collisions would be much longer than the time scale for any photo-physical process to occur.

In this paper, the photo-physical properties of R575 cations when irradiated with a single nanosecond laser pulse are examined via action-spectroscopy in both visible and ultra-violet. The results are compared to the calculated transition energies from TDDFT and the nature of the transitions are discussed. These results may help to clarify the large errors that are often found in the calculation of the $\mathrm{S}_{0} \rightarrow \mathrm{S}_{1}$ transition energy of rhodamine dyes. The origin of the two photon dependence of the $S_{1}$ state and the failure of action spectroscopy to recover an accurate value for the absorption maximum are explained in terms of a $1+1$ photofragmentation scheme where the second photon is absorbed from the $\mathrm{S}_{1}$ to a higher lying singlet state from which fragmentation can occur. A 1+1' photo-fragmentation scheme is also presented in which the total energy of the two photons are maintained whilst the individual photon energies are varied allowing a reproduction of the $S_{1}$ absorption maximum via action spectroscopy. 
2 Method.

\subsection{Experimental.}

$5 \mathrm{mg}$ of 5-carboxyrhodamine $575 \mathrm{C}_{5}$-maleimide (Setareh Biotech), shown in figure 1, was dissolved in $500 \mu \mathrm{DMSO}$, before being subsequently diluted to a concentration of $20 \mu \mathrm{M}$ in 1:1 $\mathrm{H}_{2} \mathrm{O}: \mathrm{CH}_{3} \mathrm{OH} 0.1 \%$ acetic acid for use in the electrospray source.

The experimental setup used to perform the experiments presented in this paper has been described in detail previously. ${ }^{32}$ Briefly, a linear quadrupole ion trap mass spectrometer (LTQ Velos, Thermo Fisher Scientific, San Jose, CA) was used to generate, mass select and trap ions in a first, high pressure ion trap for a controlled duration. During ion trapping, ions can be activated and fragmented by collisions (CID) or photons (LID). Fragment ions are transmitted to a second ion trap, with a low pressure, where they are mass analyzed. A fused silica window ( $3 \mathrm{~mm}$ thick, 1 inch diameter) is positioned at the back end of the instrument and allows for the introduction of the lasers in the UV-Visible range along the axis of the linear ion traps. 1-2 $\mathrm{mm}$ diameter circular openings in trapping-electrodes enable the interaction of the trapped ions with an on axis laser beam. In order to optimize laser transmission, the central hole of the electrode closest to the fused silica window was enlarged to $5 \mathrm{~mm}$ in diameter. 


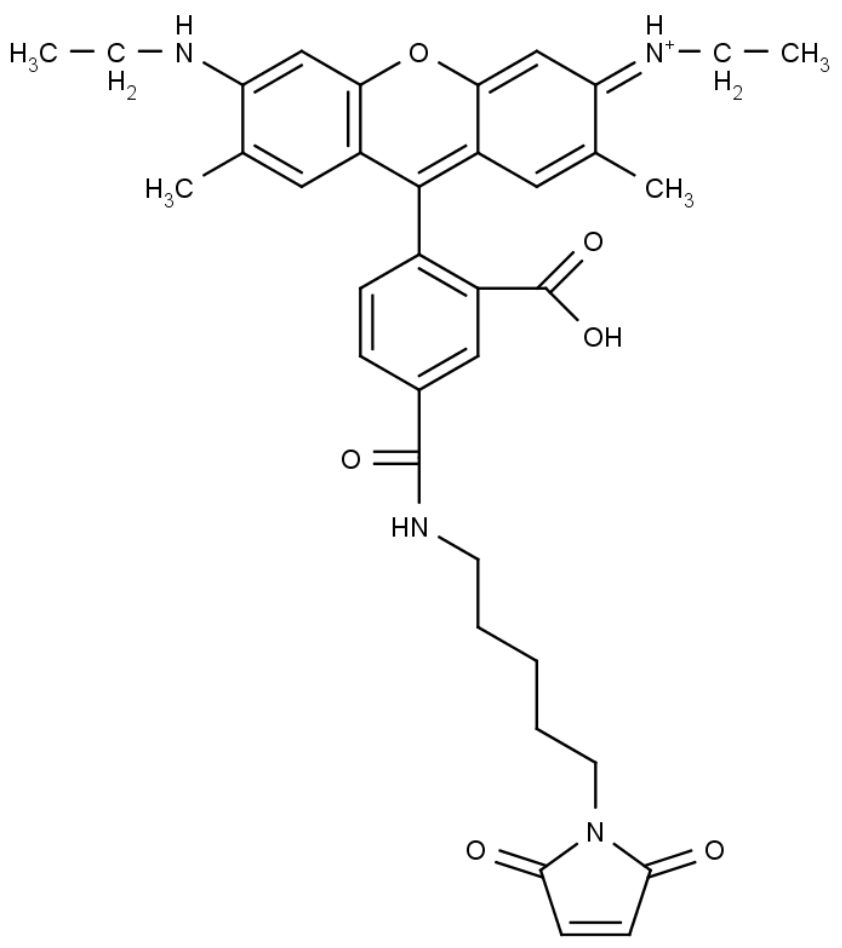

Figure 1. The structure of carboxyrhodamine $575 \mathrm{C}_{5}$-maleimide.

For 1-colour experiments, the light source used is a Panther EX OPO pumped by the third harmonic $(355 \mathrm{~nm})$ of a Surelite II Nd:YAG laser (Continuum, Santa Clara, CA). A repetition rate of $10 \mathrm{~Hz}$ and pulse-widths of the order of $5 \mathrm{~ns}$ were used. For visible spectra, the visible portion of the spectrum was used directly via the signal beam of the OPO $(410-700 \mathrm{~nm})$. For UV spectra, the signal beam of the OPO was doubled to give wavelengths between $215-350$ $\mathrm{nm}$. Pulse energies of between 1.0 and $4.0 \mathrm{~mJ} /$ pulse were used, and the beam was focused into the first ion trap by a $1000 \mathrm{~mm}$ focal length convergent lens in both cases. A mechanical shutter, synchronized with the mass spectrometer, is used to stop the beam at all times except the 'ion activation window' - that is the time after ion accumulation and before the mass analysis. A single laser pulse was used for the irradiation of the trapped ions and when irradiating ions the normalized collision energy is kept at zero. LID mass spectra were recorded for a period of 1 minute at each wavelength. The power was monitored using a power meter (Ophir-Spiricon GmbH, Ahrensburg, Germany), and was taken as the average 
value measured for a duration of one minute directly after the mass spectrum accumulation had taken place.

For two colour experiments, the pump laser was the Panther EX OPO as described above. The probe laser was a Horizon OPO pumped by the third harmonic of a second Surelite I Nd:YAG laser. The probe laser was triggered by the pump laser to ensure synchronous arrival of the two laser pulses into the first ion trap. A mechanical shutter was placed in each beam, and both were synchronized with the mass spectrometer so as to only allow light to pass into the ion trap during the ion activation window as described above. The pump and probe beams were combined using a $505 \mathrm{~nm}$ long pass dichroic mirror, and subsequently passed through a $1000 \mathrm{~mm}$ focal length lens into the ion trap. Wavelengths of between $460-505 \mathrm{~nm}$ for the pump and 535 - $597 \mathrm{~nm}$ for the probe were used. The power of each laser was measured individually using the power meter immediately after the dichroic mirror. At each pump and probe wavelength, three mass spectra were recorded; pump only, probe only and pump + probe. The former two were subtracted from the latter to give the fragmentation efficiency due to the pump + probe only.

\subsection{Computational}

Geometry optimizations and the calculation of ground and excited state properties were performed in the frame of density functional theory (DFT). The global hybrid functional B3LYP with a 6-31+G** basis set was used, and Grimme's D3 dispersion correction with Becke-Johnson damping was included for the optimization of structures. ${ }^{33-36}$ For the timedependent DFT (TDDFT) calculation of excited states, the $\omega$ B97X functional was used, as it has been benchmarked to be accurate for a broad range of organic molecules. ${ }^{37-39}$ Here, a bigger basis set $\left(6-311++\mathrm{G}^{* *}\right)$ was also used. All calculations employed the Gaussian09 Revision D.01 suite of programs. ${ }^{40}$ Semi-empirical PM7 calculations were conducted using the MOPAC2012 code. ${ }^{41,42}$ Molecular Dynamics employed the Velocity-Verlet algorithm of a 
local code with 1 fs time-step totalling to $1 \cdot 10^{5}$ steps. Thermalization was achieved by coupling to a heat-bath with the Berendsen scheme (200 steps lag time).

The thermal broadening is based on a canonical ensemble of structures which were obtained using constant-temperature molecular dynamics with the semi-empirical PM7 Hamiltonian. From the 100 ps trajectory, 99 structures (every $1000^{\text {th }}$ step) were extracted and their vertical absorption spectrum computed at the $\omega \mathrm{B} 97 \mathrm{XD} / 6-31+\mathrm{G}^{* *}$ level of theory. The resulting simulated spectra for each structure were combined to obtain the final $300 \mathrm{~K}$ absorption spectrum.

\section{Results and discussion}

The gas phase absorption spectrum of R575 has been measured in the visible region previously by both action spectroscopy and laser induced fluorescence. To the best of the authors knowledge there have been no UV spectrum reported for any rhodamine dye in the gas phase. The action-spectrum of R575 is shown in the top panel of figure 2 between $220 \mathrm{~nm}$ and $590 \mathrm{~nm}$, with 3 bands observed. The bottom panel of figure 2 contains the simulated absorption spectrum obtained with TDDFT. There is an excellent agreement between experimental and calculated spectra observed in the UV region of the spectrum (bands B and C), but the agreement is noticeably worse in the visible region (band A).

It has been previously noted that the $\mathrm{S}_{1}$ transition of $\mathrm{R} 575$ is not well recovered by action spectroscopy, a fact that was attributed to the high fluorescence quantum yield of this molecule - and hence a low quantum yield for non-radiative relaxation and hence fragmentation following internal conversion. ${ }^{22}$ Previous studies of R575 using laser induced fluorescence placed the absorption maximum at $495 \mathrm{~nm}$, whilst a study of R575 doubly grafted onto a small tripeptide to quench fluorescence by forced proximity of the dyes shows a maximum at $505 \mathrm{~nm}^{23,28}$ Figure 3(a), which gives the dependence of the fragmentation yield as a function of laser power for the $S_{1}$ transition, shows there is a clear quadratic 
dependence, implying a two-photon photo-fragmentation process must occur. This is in agreement with previous measurements of the power dependence of fragmentation from the $\mathrm{S}_{1}$ state. ${ }^{25-27}$ These studies have all used either continuous or high repetition-rate lasers. The concurrent absorption of two photons - i.e. before any relaxation process could occur - was considered unlikely as compared to the sequential absorption when using such lasers. This conclusion was supported by the observation that photo-fragmentation was sensitive not only to the laser fluence but also to the pressure of the buffer gas. ${ }^{25-27}$ Thus the multi-photon dependence was explained by either internal conversion or intersystem crossing followed by absorption of a second photon which reached some dissociative state.

However, in this work only a single nanosecond pulse was used to induce photofragmentation and thus any two-photon dependence can only be explained by the absorption of two photons within the $5 \mathrm{~ns}$ width of the laser pulse. This timescale is much shorter than the average time between collisions - which is approximately $50 \mu \mathrm{s}$ for the pressure of gas present in our ion trap - and thus the influence of collision-induced internal-conversion or intersystem crossing can be discounted. Thus, it seems reasonable that the absorption of the second photon occurs directly from the $S_{1}$ state to a higher lying singlet state. This supposition is in agreement with the measured fluorescence lifetime of $5.65 \mathrm{~ns}$ for gas phase R575 cations, which is of the same order as the pulse width of the nanosecond lasers used here. ${ }^{31}$ It can therefore be expected that during irradiation there is a significant population of R575 in the $\mathrm{S}_{1}$ electronic state at any given time available to absorb a second photon. 


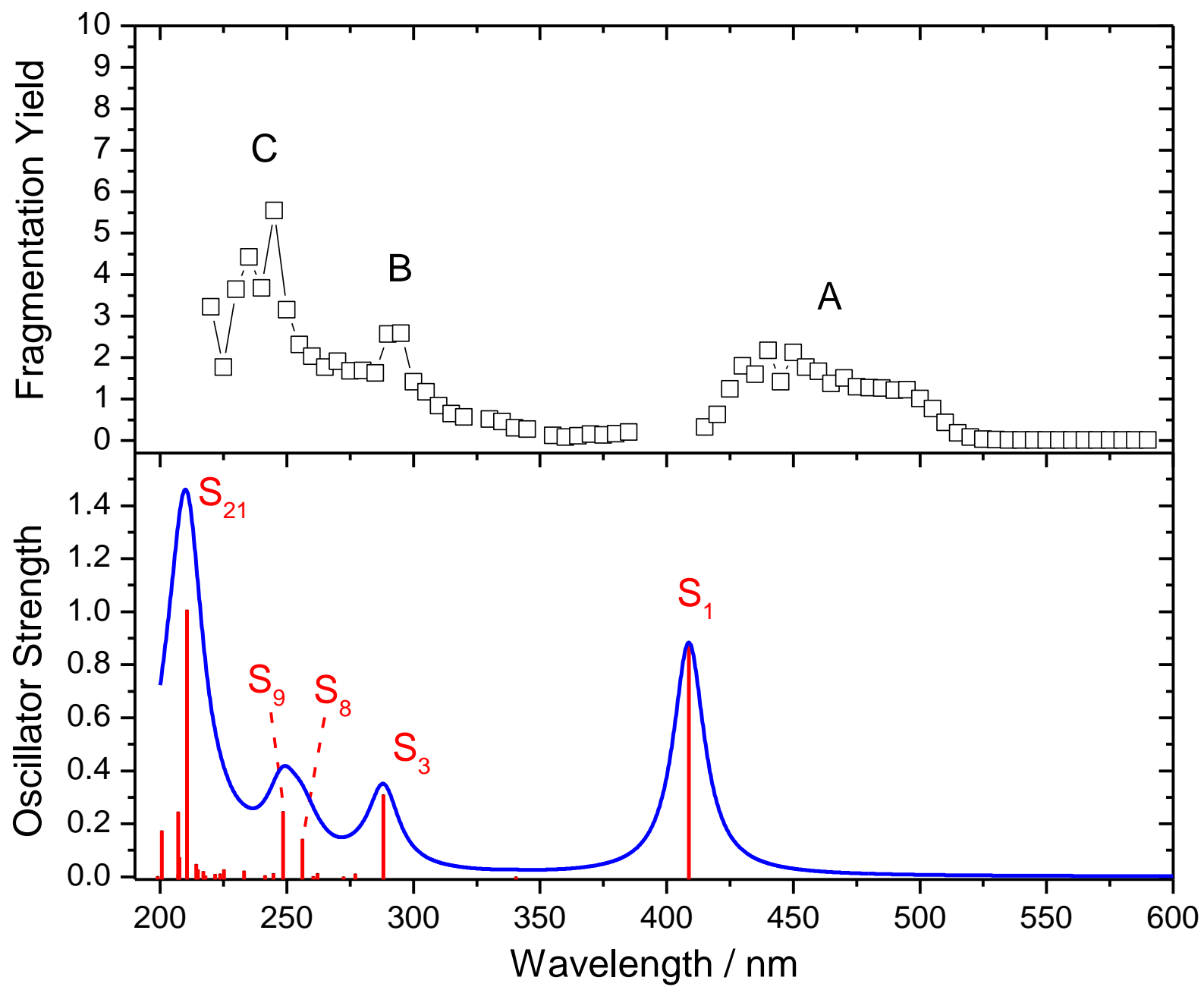

Figure 2. Experimental action spectrum (top panel) and theoretical vertical absorption spectrum (bottom panel, red sticks) convoluted with a Lorentzian line-shape functions of a half-widths of $15 \mathrm{~nm}$ (bottom panel, blue curve) for R575. The red labels show the main calculation transitions, whilst the black labels denote the experimentally observed bands.

The two-photon nature of the photo-fragmentation from the $S_{1}$ state can also explain why the expected absorption maximum is not recovered using action spectroscopy. The measurement being performed when the fragmentation efficiency of the $\mathrm{S}_{1}$ absorption is monitored is a $1+1$ photo-fragmentation and as such will depend not just on the absorption to the $\mathrm{S}_{1}$ but also on the properties of the higher lying singlet state from which the observed fragmentation occurs, 
see the left hand side of figure 4. R575 is found to absorb between 520 and $415 \mathrm{~nm}$, and thus due to the $1+1$ photo-fragmentation scheme in these experiments this is equivalent energetically - to absorption of a single photon between 260 and $207.5 \mathrm{~nm}$ respectively. Comparison with the UV spectrum in figure 2 for this wavelength range shows that this in in the region of the $\mathrm{C}$ band, found at $\sim 240 \mathrm{~nm}$. Thus, the increase in fragmentation efficiency and observed maximum of $460 \mathrm{~nm}$ for the $\mathrm{S}_{1}$ absorption can be explained, at least partially, by there being the most efficient $1+1$ absorption leading to photo-fragmentation between $S_{1}$ and the higher lying singlet state rather than being the maximum of absorption in the $S_{1}$.

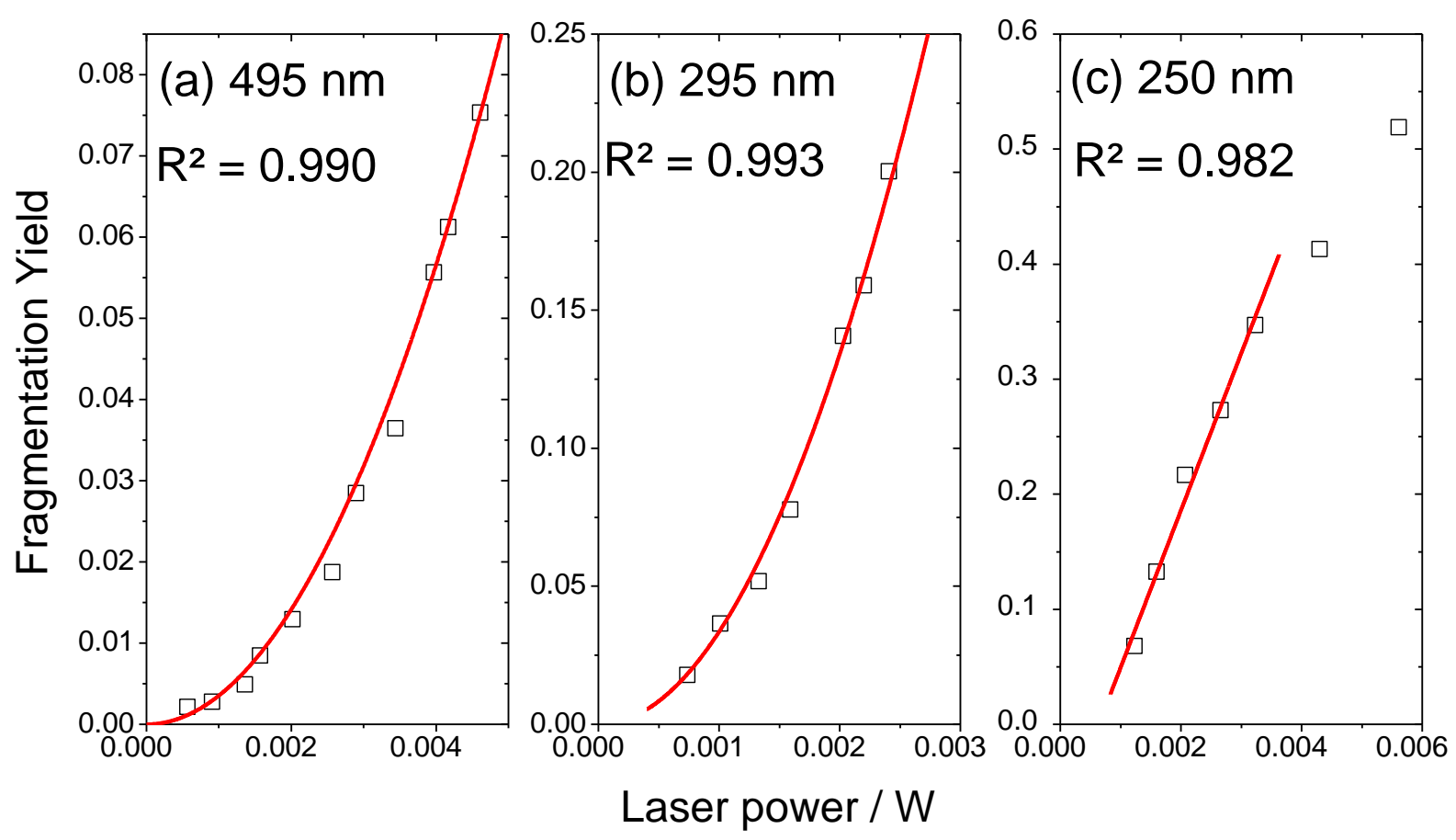

Figure 3. Dependence of the fragmentation yield as a function of power at (a) $495 \mathrm{~nm}$, (b) 295 $\mathrm{nm}$ and (c) $240 \mathrm{~nm}$. The red curve represents a fit to a second order polynomial for panels (a) and (b), and a straight line for panel (c), with the associated $\mathrm{R}^{2}$ value indicated. The errors in a single measurement of the fragmentation yield, as calculated by Poisson statistics, is smaller than the size of the data point and are thus omitted. 


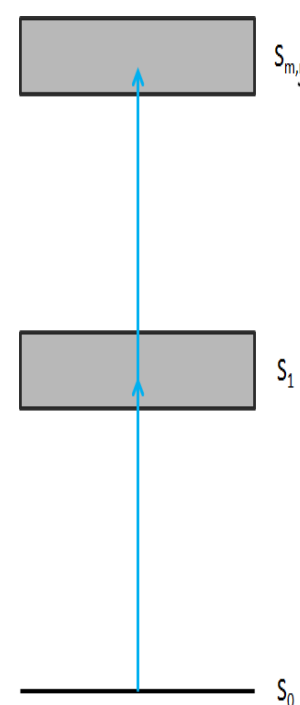

1-colour scheme

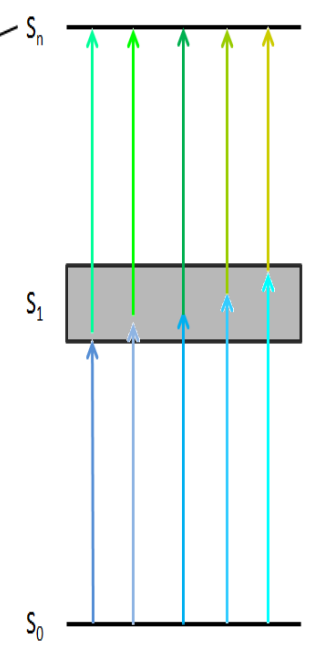

2-colour scheme

Figure 4. Schematic representation of one-colour (left) and two-colour (right) schemes employed in this work to study the fragmentation after absorption of a photon by the $\mathrm{S}_{1}$ state of R575 cations.

The nature of the photo-fragmentation suggests an alternative method for probing the properties of the $\mathrm{S}_{1}$ directly, using a $1+1$ ' absorption scheme. In such an experiment, two photons of different wavelengths are used - the first to pump R575 to the $S_{1}$ state and the second to excite to the higher lying singlet state that leads to dissociation, see the right hand side of figure 4 . The total energy of the two photons - and thus the total energy added to the system - is kept constant when the wavelengths of the pump and probe photon are changed such that fragmentation happens after absorption to the same higher-lying singlet state. The probe laser wavelengths were chosen such that the photon energy was smaller than the $S_{1}$ transition energy, and thus absorption of a probe photon by ground state R575 was not possible. In this scheme, the amount of two colour photo-fragmentation observed will depend only on the population of $\mathrm{R} 575$ in the $\mathrm{S}_{1}$ state since the probe photon has an energy below that of the $S_{1}$ transition energy. Such a measurement can be obtained by measurement of the fragmentation yield with both pump and probe laser, and subtracting the value of the 
fragmentation yield of pump and probe lasers alone (see figure S1 in the supporting information).

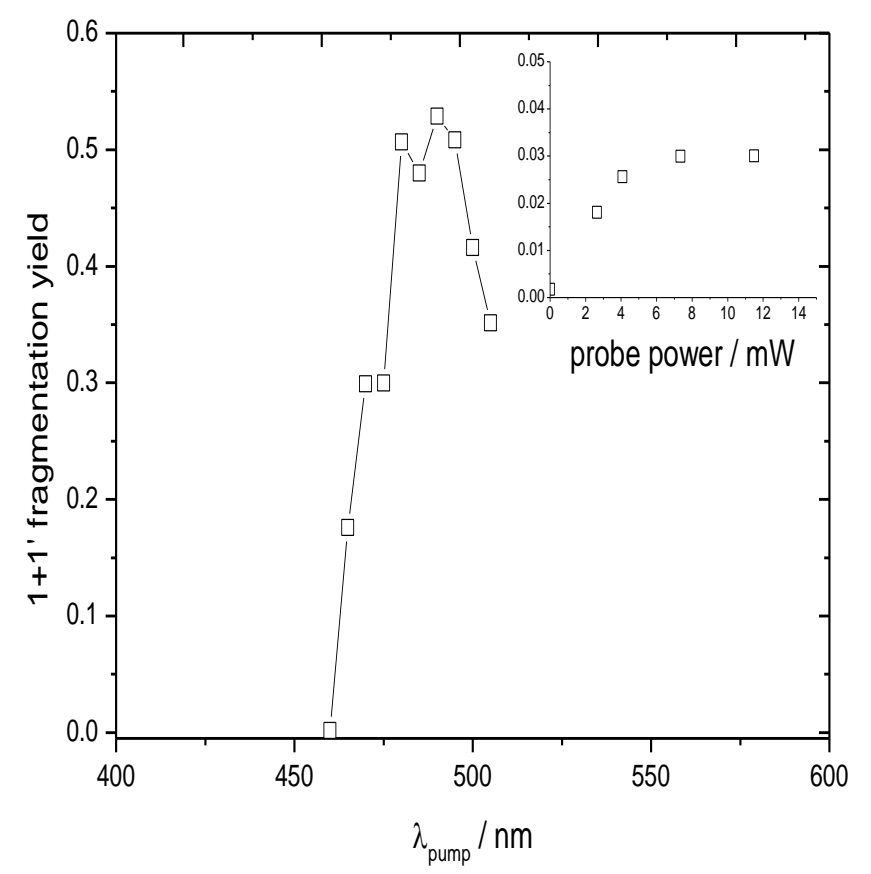

Figure 5. 1+1' fragmentation yield as a function of the pump wavelength for a fixed total energy. The inset shows the dependence of the $1+1^{\prime}$ corrected fragmentation yield on the power of the probe laser for a fixed pump laser power of $1 \mathrm{~mW}$.

Figure 5 shows the 2-colour fragmentation yield as a function of the probe photon wavelength. For the measurements performed here, a total energy for the two laser pulses of $4.772 \mathrm{eV}$ was used, with pump laser wavelengths ranging from 460 - $505 \mathrm{~nm}$ and probe laser wavelengths from $535-596.86 \mathrm{~nm}$. The fragmentation efficiencies for both pump and probe laser alone were also monitored (see figure S2), confirming that it was not possible for the probe laser to be absorbed from $\mathrm{R} 575$ in its $\mathrm{S}_{0}$ state. A peak centred at $490 \mathrm{~nm}$ is observed in the two-colour spectrum of figure 5. The difference observed between the spectrum in figure 2 and figure 5 shows that the shoulder observed at $\sim 440 \mathrm{~nm}$ is due to 2-photon absorption, and that the true $S_{1}$ absorption of R575 corresponds to the band at $490 \mathrm{~nm}$ in figure 5.This 
value is slightly lower than the $505 \mathrm{~nm}$ observed by action-spectroscopy of pseudo-dimers of R575 and $495 \mathrm{~nm}$ measured by laser induced fluorescence (LIF). ${ }^{22,} 27$ Such small differences are expected when comparing LIF and action-spectroscopic methods for determining the real maximum of absorption, and shifts in the transition energy are expected in dimeric systems. The inset of figure 5 shows the 2-colour fragmentation as a function of the probe laser power, which shows an initial linear increase $\left(\mathrm{R}^{2}=0.997\right)$ followed by a plateau corresponding to saturation of the irradiated ions. This confirms that it is absorption of a single photon from R575 cations electronically excited into the $S_{1}$ state that is causing the increased 2-colour fragmentation yield. Since it is expected that such a 2-photon photo-fragmentation scheme will be observed in other fluorescent rhodamine derivatives, this method provides a universal way to accurately probe the $S_{1}$ state via photo-fragmentation spectroscopy.

The value of $490 \mathrm{~nm}$ for the $\mathrm{S}_{1}$ transition of R575 is significantly different to the value of 409 nm predicted by TDDFT. This is a well-known problem for such xanthene based dyes, with calculations of $S_{1}$ transition energies of various rhodamine derivatives showing a systematic over-estimation of the transition energy by as much as $0.6 \mathrm{eV} .^{43}$ Although the use of a global hybrid density functional with exact Hartree-Fock exchange and a long-range correction is expected to alleviate poor charge-transfer descriptions in many molecules, the $\mathrm{S}_{1}$ state seems still to suffer from a strong (blue) shift as compared to experiment. Previously reported calculations employing a variety of density functionals overestimate the rhodamine absorption band peak of about $0.4 \mathrm{eV}$ as a general trend to the TDDFT method for this type of dyes. ${ }^{43-46}$ The possible origins of the deviation between TDDFT and experiment may lie in negligence of electron correlation or non-verticality of the absorption (due to strong vibronic coupling). ${ }^{39}$ Additionally, thermal effects may influence the position of the absorption maxima as compared to the often used $0 \mathrm{~K}$ approximation when TDDFT spectra are considered. In the following, these three factors are disentangled by separately considering explicit thermal 
broadening of the absorption spectrum, potential impact of vibronic coupling within the TDDFT approach and - qualitatively - the impact of electron correlation using semiempirical multi-electron configurational calculations.

From the $0 \mathrm{~K}$ and $300 \mathrm{~K}$ spectrum, shown in figure $\mathrm{S} 3$, a shift of $-0.1 \mathrm{eV}$ in the $300 \mathrm{~K}$ spectrum with respect to $0 \mathrm{~K}$ is evident. Based on this analysis we propose a thermochromic redshift for the $\mathrm{S}_{1}$ of about $25 \mathrm{~nm}$ with regards to the $0 \mathrm{~K}$ prediction.

The calculation of excited state relaxation gave access to an estimate of the maximal possible redshift of the transition due to non-verticality of the absorption. The adiabatic $0-0$ transition is calculated to occur at a wavelength of $411 \mathrm{~nm}$. In accordance to the known small Stokes shift of R575 in the gas-phase (11 nm as measured by Talbot et al.), this contribution is considered to be less than $0.05 \mathrm{eV}$ (about $10 \mathrm{~nm}$ in the region between 400 and $500 \mathrm{~nm}$ ). ${ }^{19}$

Finally, in order to obtain a rough estimate of the importance of electron correlation, We employ a systematic semi-empirical multi-electron configuration interaction calculation using the PM7 Hamiltonian. The $S_{1}$ excitation energies using active spaces of 2 to 8 electrons in 2 to 9 levels (denoted as 2,2 to 8,9) are given in table S1 and figure S4 in the supporting information. According to these calculations, the effect of correlating up to about 15,000 configurations can be as much as $0.8 \mathrm{eV}$ within this model. Hence, it can be concluded that electron correlation may be the most important influential factor for the observed deviation between TDDFT and experiment. While we can quantify a thermochromic shift of about 25 $\mathrm{nm}$ to be present apart from the deficient electron correlation description, only a small vibronic shift is held responsible for the deviation between TDDFT and experimental $S_{1}$ absorption energies of R575 as reported in figure 2, and potentially in xanthene-based dyes in general. 
Considering now bands $\mathrm{B}$ and $\mathrm{C}$, comparison to the simulated spectrum indicates that these to be the $S_{3}$ and a combination of the $S_{8}$ and $S_{9}$ transitions respectively (see figure 2). In fact, the agreement of the TDDFT and experimental spectra is much better than for the $\mathrm{S}_{1}$ state and motivates an attempt assign the nature of the excited states according to the analysis of the excitations. It should also be noted that in the experimental spectrum, a shoulder is observed at $330 \mathrm{~nm}$. Indeed the TDDFT calculation predicts the $\mathrm{S}_{2}$ state to lie at an energy of $3.68 \mathrm{eV}$ $(337 \mathrm{~nm})$ and to be optically dark (oscillator strength $\left.\mathrm{f}_{\mathrm{e}}=0.001\right)$.

The evolution of fragmentation yield as a function of laser power for band B and band C are shown in figure 3 (b) and (c) respectively. Band $\mathrm{C}$ has an initially linear relationship, which indicates fragmentation is via absorption of a single photon. Above $3 \mathrm{~mW}$, a deviation from this linear relationship is observed which indicates the onset of saturation. For band B, a quadratic relationship is observed, which is indicative of a 2-photon absorption process similar to that observed for the $S_{1}$ transition. To understand the physical reason behind the fragmentation behaviour, it is instructive to consider the nature of the transitions found by TDDFT. This analysis is detailed in figure 6 by visualizing the leading Kohn-Sham orbital excitations for $S_{1}$ and $S_{3}$ as well as excited-ground state electron density difference plots for $\mathrm{S}_{8}, \mathrm{~S}_{9}$ and $\mathrm{S}_{21}$ (see also supplementary material table $\mathrm{S} 2$ and Figure $\mathrm{S} 5$ ). The $\mathrm{S}_{1}$ state is mainly composed of the HOMO-LUMO excitation, while $\mathrm{S}_{3}$ mainly consists of two excitations with $\pi-\pi^{*}$ character (occupied orbitals being the HOMO-1 and HOMO-2) and a (minor) charge transfer type of excitation from the carboxyphenyl moiety to the xanthene $\pi$ system. This renders the $S_{1}$ and $S_{3}$ transitions "local" in character.

The higher-energy transitions are comprised of a more complicated character with a significant number of one-electron excitations contributing. For this reason we depict the overall electron charge redistribution upon the optical transition from the difference between relaxed excited state density and ground state electronic density. $S_{8}$ and $S_{9}$ are very similar 
and consist of a mixture between xanthene $\pi-\pi^{*}$ and $n-\pi^{*}$ excitation within the carboxyphenyl moiety. This "non-local" character is in contrast to the $\mathrm{S}_{1}$ and $\mathrm{S}_{3}$ transitions (notice that the use of a hybrid long-range-corrected functional should not suffer from charge-transfer "contamination" as do pure density functionals). ${ }^{47}$ The intense transition $\mathrm{S}_{21}$ is again almost entirely due to a xanthene $\pi-\pi^{*}$ excitation with very little contribution of charge transfer or $n$ $\pi^{*}$ excitation, giving it similar local character to the $\mathrm{S}_{1}$ and $\mathrm{S}_{3}$ transitions.
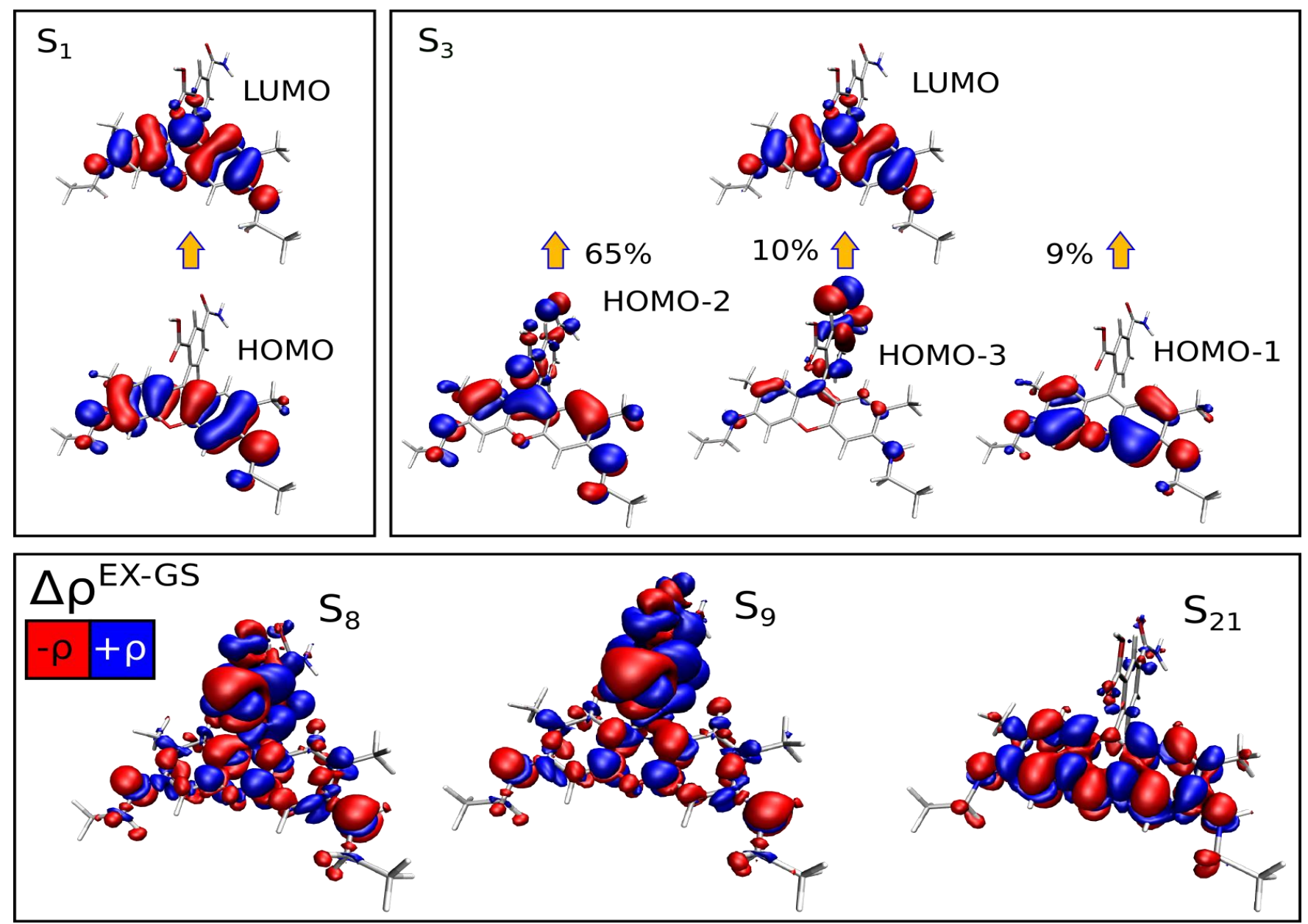

Figure 6. The leading Kohn-Sham orbitals found from the TDDFT calculations for the $\mathrm{S}_{1}$ and

$\mathrm{S}_{3}$ (top two panels) as well as the excited-ground state electron density difference plots for the $\mathrm{S}_{8}, \mathrm{~S}_{9}$ and $\mathrm{S}_{21}$

A further point to consider is that the primary fragmentation channels of R575 all involve cleavage of a $\mathrm{C}-\mathrm{C}$ bond, which requires an energy of $\sim 3.6 \mathrm{eV}$. This is $\sim 1 \mathrm{eV}$ higher than band $\mathrm{A}$ and thus a 1-photon fragmentation is not energetically possible. Bands $\mathrm{B}$ and $\mathrm{C}$ both 
lie above this energy, and thus their different photo-fragmentation behaviour may lie within the different nature of the transitions involved.

Thus, a tentative explanation for the observed photon dependences of the different bands can be given. Band A possesses a 2-photon dependence as the transition energy for the $\mathrm{S}_{1}$ state is below the expected bond dissociation energy for the major fragmentation pathways. For band $\mathrm{B}$, the local nature of the $\mathrm{S}_{3}$ excitation conveys a short radiative lifetime to the state. Hence, non-radiative relaxation is less important and it is absorption of a second photon to a higher lying state that gives most of the observed fragmentation. Finally, the $\mathrm{S}_{8}$ and $\mathrm{S}_{9}$ excitations that comprise band $\mathrm{C}$ possesses significant non-local character, which has been linked with higher non-radiative relaxation and hence a higher fragmentation efficiency. This influence of charge-transfer like transitions on the relaxation properties of the excited states of R575 is consistent with the work of Savarese et al., who found radiative lifetime to be dependent on the amount of phenyl to xanthene charge transfer in the $\mathrm{S}_{0} \leftarrow \mathrm{S}_{1}$ deactivation in a closely related dye. ${ }^{43}$

\section{Conclusion}

The absorption of rhodamine 575 cations has been studied using action spectroscopy between 220 and $590 \mathrm{~nm}$ and compared to calculated transition energies from TDDFT. In the visible region, the failure to recover the expected absorption maximum was rationalised in terms of the two photon dependence, leading to $1+1$ photo-fragmentation via absorption of a first photon taking R575 to the $\mathrm{S}_{1}$ state followed by absorption of a second photon from the $\mathrm{S}_{1}$ state to a higher lying singlet state from which dissociation could occur. A 1+1' photofragmentation scheme, where the total energy of the two photons was kept constant to ensure excitation occurs from the same point on the excited singlet state, but where the energy of each photon was changed allowed recovery of the $S_{1}$ absorption maximum at $490 \mathrm{~nm}$, which is in good agreement with previous measurements. Such a 1+1' fragmentation scheme would 
be applicable to any highly fluorescent rhodamine derivative, which have similar properties to R575 in the gas phase.

The difference in observed power dependence for the different bands observed experimentally was tentatively explained in terms of the nature of the excitations that contribute to them. The local nature of the $\mathrm{S}_{1}$ and $\mathrm{S}_{3}$ states leads to a short radiative lifetime and thus absorption of a second-photon to a higher lying state was required to measure any photo-fragmentation, resulting in a quadratic dependence of photo-fragmentation yield on laser power. Additionally, the $\mathrm{S}_{1}$ state has a transition energy below that of the expected bond dissociation energy for the major fragmentation pathways. In contrast, the non-local nature of the $\mathrm{S}_{8}$ and $\mathrm{S}_{9}$ transitions allows access to a more diverse range of deactivation pathways, including direct photo-fragmentation. This competition between local and non-local character of excitations is as theorized by Savarese et al. ${ }^{43}$

From our theoretical work complementing the $0 \mathrm{~K}$ vertical TDDFT absorption spectrum, we have gained insight into the factors that lead to the significant overestimation of the $S_{1}$ transition energy R575. We considered thermochromic and possible vibronic shifts and qualitatively - electron correlation effects. It was found that the main contribution to this failure is due to the latter but a most probably smaller, but significant effect of as much as 25 $\mathrm{nm}$ arises from thermochromism at $300 \mathrm{~K}$. Vibronic effects were found to play only a minor role. We would like to emphasize that in view of our focus on the UV part of the spectrum we suggest that performing higher-level ab-initio calculations on the $S_{1}$ state in order to better quantify electron correlation versus thermochromic and vibronic shifts. 


\section{ASSOCIATED CONTENT}

Mass spectra for pump only, probe and pump+probe LID experiments, 1 color action spectra for pump and probe wavelengths only, and further details of the thermochromism and electronic configurations can be found in the supplementary information. This material is available free of charge via the Internet at http://pubs.acs.org.

\section{AUTHOR INFORMATION}

\section{Corresponding Author}

* To whom correspondence should be addressed. Email: philippe.dugourd@univ-lyon1.fr

\section{Author Contributions}

All authors have given approval to the final version of the manuscript.

\section{Funding Sources}

The research leading to these results has received funding from the European Research Council under the European Union's Seventh Framework Programme (FP7/2007-2013 Grant agreement $\mathrm{N}^{\circ}$ 320659). A.K. acknowledges funding from the Deutsche Forschungsgemeinschaft DFG (Research Fellowship Ku 3251/1-1)

\section{ACKNOWLEDGMENT}

We gratefully acknowledge computer time granted by the PSMN (Ple Scientifique de Modélisation Numérique) computing center of ENS de Lyon. 


\section{REFERENCES}

1. Arbeloa, F. L.; Arbeloa, T. L.: Estevez, M. J. T.; Arbeloa, I. L. Photophysics of Rhodamines - Molecular-Structure and Solvent Effects. J. Phys. Chem.1991, 95, 2203-2208.

2. Arbeloa, T. L.; Arbeloa, F. L.; Bartolome, P. H.; Arbeloa, I. L. On the Mechanism of Radiationless Seactivation of Rhodamines. Chem. Phys.1992, 160, 123-130.

3. Arbeloa, F. L. ; Arbeloa, T. L.; Lage, E. G.; Arbeloa, I. L.; Deschryver, F. C. Photophysical Properties of Rhodamines with Monoethylamino Group-r19 and Group-r6g in Water Ethanol Mixtures. J. Photoch. Photobio. A 1991, 56, 313-32.

4. Arbeloa, T. L.; Estevez, M. J. T.; Arbeloa, F. L.; Aguirresacona, I. U.; Arbeloa, I. L.; Luminescence Properties of Rhodamines in Water-Ethanol Mixtures. J. Lumin. 1991, 48-49, 400-404.

5. Arden, J.; Deltau, G.; Huth, V.; Kringel, U.; Peros D.; Drexhage, K. H.; Fluorescence and Lasing Properties of Rhodamine Dyes. J. Lumin. 1991, 48-49, 352-358.

6. Barigelletti, F.; Effect of Temperature on the Photophysics of Rhodamine-101 in a Polar-Solvent. Chem. Phys. Lett. 1987, 140, 603-606.

7. Beaumont, P. C.; Johnson D. G.; Parsons, B. J. Photophysical Properties of Laser-Dyes

- Picosecond Laser Flash-Photolysis Studies of Rhodamine-6G, Rhodamine-B and Rhodamine-101. J. Chem. Soc. Faraday T. 1993, 89, 4185-4191.

8. Ferguson, M. W.; Beaumont, P. C.; Jones, S. E.; Navaratnam S.; and Parsons, B. J. Excited State and Free Radical Properties of Rhodamine 123: a Laser Flash Photolysis and Radiolysis Study. Phys. Chem. Chem. Phys. 1999, 1, 261-268.

9. Karstens T.; Kobs, K. Rhodamine-B and Rhodamine-101 as Reference Substances for Fluorescence Quantum Yield Measurements. J. Phys. Chem. 1980, 84, 1871-1872.

10. Magde, D.; Rojas, G. E.; Seybold, P. G. Solvent Dependence of the Fluorescence Lifetimes of Xanthene Dyes. Photochem. Photobiol. 1999, 70, 737-744. 
11. Snare, M. J.; Treloar, F. E.; Ghiggino, K. P.; Thistlethwaite, P. J. The Photophysics of Rhodamine-B. J. Photochem. 1982, 18, 335-346.

12. Chingin, K.; Chen, H. W.; Gamez, G.; Zenobi, R. Exploring Fluorescence and Fragmentation of Ions Produced by Electrospray Ionization in Ultrahigh Vacuum. J. Am. Soc. Mass Spectr. 2009, 20, 1731-1738.

13. Clemen, M.; Gernert, C.; Peters J.; Grotemeyer, J. Fragmentation Reactions of Labeled and Unlabeled Rhodamine B in a High-Resolution Fourier Transform Ion Cyclotron Resonance Mass Spectrometer. Eur. J. Mass Spetrom.2013, 19, 135-139.

14. Peters, J.; Clemen M.; Grotemeyer, J. Fragmentation of Deuterated Rhodamine B Derivates by Laser and Collisional Activation in an FT-ICR Mass Spectrometer. Anal. Bioanal. Chem. 2013, 405, 7061-7069.

15. Frankevich, V.; Guan, X. W.; Dashtiev M.; Zenobi, R.; Laser-induced Fluoresence of Trapped Gas-Phase Molecular Ions Generated by Internal-source Matrix-Assisted Laser Desorption/Ionization in a Fourier Transform Ion Cyclotron Resonance Mass Spectrometer. Eur. J. Mass Spectrom. 2005, 11, 475-482.

16. Frankevich, V.; Sinues, P. M. L.; Barylyuk, K.; Zenobi, R.; Ion Mobility Spectrometry Coupled to Laser-Induced Fluorescence. Anal. Chem. 2013, 85, 39-43.

17. Peters, J.; Grotemeyer, J.; Fragmentation of Xanthene Dyes by Laser Activation and Collision-Induced Dissociation on a High-resolution Fourier Transform Ion Cyclotron Resonance Mass Spectrometer. Rapid. Commun. Mass Sp. 2011, 25, 1169-1172.

18. Sagoo, S. K.; Jockusch, R. A.; The Fluorescence Properties of Cationic Rhodamine B in the Gas Phase. J. Photoch. Photobio. A 2011, 220, 173-178.

19. Talbot, F. O.; Rullo, A.; Yao H.; Jockusch, R. A. Fluorescence Resonance Energy Transfer in Gaseous, Mass-Selected Polyproline Peptides. J. Am. Chem. Soc. 2010, 132, $16156-16164$. 
20. Dashtiev, M.; Azov, V.; Frankevich, V.; Scharfenberg L.; Zenobi, R. Clear Evidence of Fluorescence Resonance Energy Transfer in Gas-Phase Ions J. Am. Soc. Mass Spectr. 2005, $16,1481-1487$.

21. Danell A. S.; Parks, J. H. FRET Measurements of Trapped Oligonucleotide Duplexes Int. J. Mass Spectrom. 2003, 229, 35-45.

22. Daly, S.; Poussigue, F.; Simon, A-L.; MacAleese, L.; Bertorelle, F.; Chirot, F.; Antoine, R.; Dugourd, P. Action-FRET: Probing the Molecular Conformation of MassSelected Gas-Phase Peptides with Forster Resonance Energy Transfer Detected by AcceptorSpecific Fragmentation. Anal. Chem. 2014, 86, 8798-8804.

23. Frankevich, V.; Chagovets, V.; Widjaja, F.; Barylyuk, K.; Yang, Z. Y.; Zenobi, R. Fluorescence Resonance Energy Transfer of Gas-Phase Ions Under Ultra-High Vacuum and Ambient Conditions. Phys. Chem. Chem. Phys. 2014, 16, 8911-8920.

24. Hendricks, N. G.; Lareau, N. M.; Stow, J. S. M.; McLean A.; Julian, R. R. BondSpecific Dissociation Following Excitation Energy Transfer for Distance Constraint Determination in the Gas Phase. J. Am. Chem. Soc. 2014, 136, 13363-13370.

25. Sassin, N. A.; Everhart, S. C.; Cline, J. I.; Ervin, K. M. Photodissociation and Collisional Cooling of Rhodamine 575 Cations in a Quadrupole Ion Trap. J. Chem. Phys. 2008, $128,234305$.

26. Sassin, N. A.; Everhart, S. C.; Dangi, K. B. B.; Ervin, M.; Cline, J. I. Fluorescence and Photodissociation of Rhodamine 575 Cations in a Quadrupole Ion Trap. J. Am. Soc. Mass Spectr. 2009, 20, 96-104.

27. Forbes, M. W.; Jockusch, R. A. Gas-Phase Fluorescence Excitation and Emission Spectroscopy of Three Xanthene Dyes (Rhodamine 575, Rhodamine 590 and Rhodamine 6G) in a Quadrupole Ion Trap Mass Spectrometer. J. Am. Soc. Mass Spectr. 2011, 22, 93-109. 
28. Chingin, K.; Balabin, R. M.;. Frankevich, V; Chen, H. W.; Barylyuk, K.; Nieckarz, R.; Fedorov A.; Zenobi, R. Optical Properties of Protonated Rhodamine 19 Isomers in Solution and in the Gas Phase. Phys. Chem. Chem. Phys. 2010, 12, 14121-14127.

29. Chingin, K.; Balabin, R. M.; Barylyuk, K.; Chen, H. W.; Frankevich V.; Zenobi, R. Rhodamines in the Gas Phase: Cations, Neutrals, Anions, and Adducts with Metal Cations. Phys. Chem. Chem. Phys. 2010, 12, 11710-11714.

30. Greisch, J. F.; Harding, M. E.; Kordel, M.; Klopper, W.; Kappes, M. M.; Schooss, D. Intrinsic Fluorescence Properties of Rhodamine Cations in Gas-Phase: Triplet Lifetimes and Dispersed Fluorescence Spectra. Phys. Chem. Chem. Phys. 2013, 15, 8162-8170.

31. Nagy, A. M.; Talbot, F. O.; Czar, M. F.; Jockusch, R. A.; Fluorescence Lifetimes of Rhodamine Dyes in Vacuo. J. Photoch. Photobio. A 2012, 244, 47-53.

32. Bellina, B.; Antoine, R.; Broyer, M.; Gell, L.; Sanader, Z.; Mitric, R.; BonacicKoutecky, V.; Dugourd, P. Formation and Characterization of Thioglycolic Acid-Silver Cluster Complexes. Dalton T. 2013, 42, 8328-8333.

33. Becke, A. D. Density-Functional Thermochemistry .3. The Role of Exact Exchange. $J$. Chem. Phys. 1993, 98, 5648-5652.

34. Lee, C. T.; Yang, W. T.; Parr, R. G. Development of the Colle-Salvetti CorrelationEnergy formula into a Functional of the Electron-Density. Phys. Rev. B 1988, 37, 785-789.

35. Stephens, P. J.; Devlin, F. J.; Chabalowski, C. F.; Frisch, M. J. Ab-initio Calculation of Vibrational Absorption and Circular-Dichroism Spectra Using Density-Functional ForceFields. J. Phys. Chem. 1994, 98, 11623-11627.

36. Grimme, S.; Ehrlich, S.; Goerigk, L. Effect of the Damping Function in Dispersion Corrected Density Functional Theory. J. Comput. Chem. 2011, 32, 1456-1465. 
37. Chai, J. D.; Head-Gordon, M. Long-Range Corrected Hybrid Density Functionals With Damped Atom-Atom Dispersion Corrections. Phys. Chem. Chem. Phys. 2011, 10, 66156620.

38. Jacquemin, D.; Perpete, E. A.; Ciofini, I.; Adamo, C. Assessment of the Omega B97 Family for Excited-State Calculations. Theor. Chem. Acc. 2011, 128, 127-136.

39. Laurent A. D.; Jacquemin, D. TD-DFT Benchmarks: A Review Int. J. Quantum Chem. 2013, 113, 2019-2039.

40. Frisch, M. J.; Trucks, G. W.; Schlegel, H. B.; Scuseria, G. E.; Robb, M. A.; Cheeseman, J. R.; Scalmani, G.; Barone, V.; Mennucci, B.; Petersson, G. A.; et al.; Gaussian 09, Revision D.01; Gaussian, Inc., Wallingford CT, 2009.

41. MOPAC2012, Stewart, J. J. P., Stewart Computational Chemistry, Colorado Springs, CO, USA, 2012.

42. Stewart, J. J. P. Ab-initio Calculation of Vibrational Absorption and CircularDichroism Spectra Using Density-Functional Force-Fields. J. Mol. Model. 2013, 19, 1-32.

43. Savarese, M.; Aliberti, A.; De Santo, I.; Battista, E.; Causa, F.; Netti, P. A.; Rega, N. Fluorescence Lifetimes and Quantum Yields of Rhodamine Derivatives: New Insights from Theory and Experiment. J. Phys. Chem. A 2012, 116, 7491-7497.

44. Pedone, A.; Bloino, J.; Monti, S.; Prampolini G.; Barone,V. Absorption and Emission UV-Vis Spectra of the TRITC Fluorophore Molecule in Solution: A Quantum Mechanical Study. Phys. Chem. Chem. Phys. 2010, 12, 1000-1006.

45. Setiawan, D.; Kazaryan, A.; Martoprawiro, M. A.; Filatov, M. A First Principles Study of Fluorescence Quenching in Rhodamine B Dimers: How Can Quenching Occur in Dimeric Species? Phys. Chem. Chem. Phys. 2010, 12, 11238-11244. 
46. Guthmuller, J.; Champagne, B. Resonance Raman Scattering of Rhodamine 6G as Calculated By Time-Dependent Density Functional Theory: Vibronic and Solvent Effects. Chemphyschem 2008, 9, 1667-1669.

47. Lange A.; Herbert, J. M. Simple Methods to Reduce Charge-Transfer Contamination in Time-Dependent Density-Functional Calculations of Clusters and Liquids. J. Chem. Theory Comput. 2007, 3,1680-1690. 

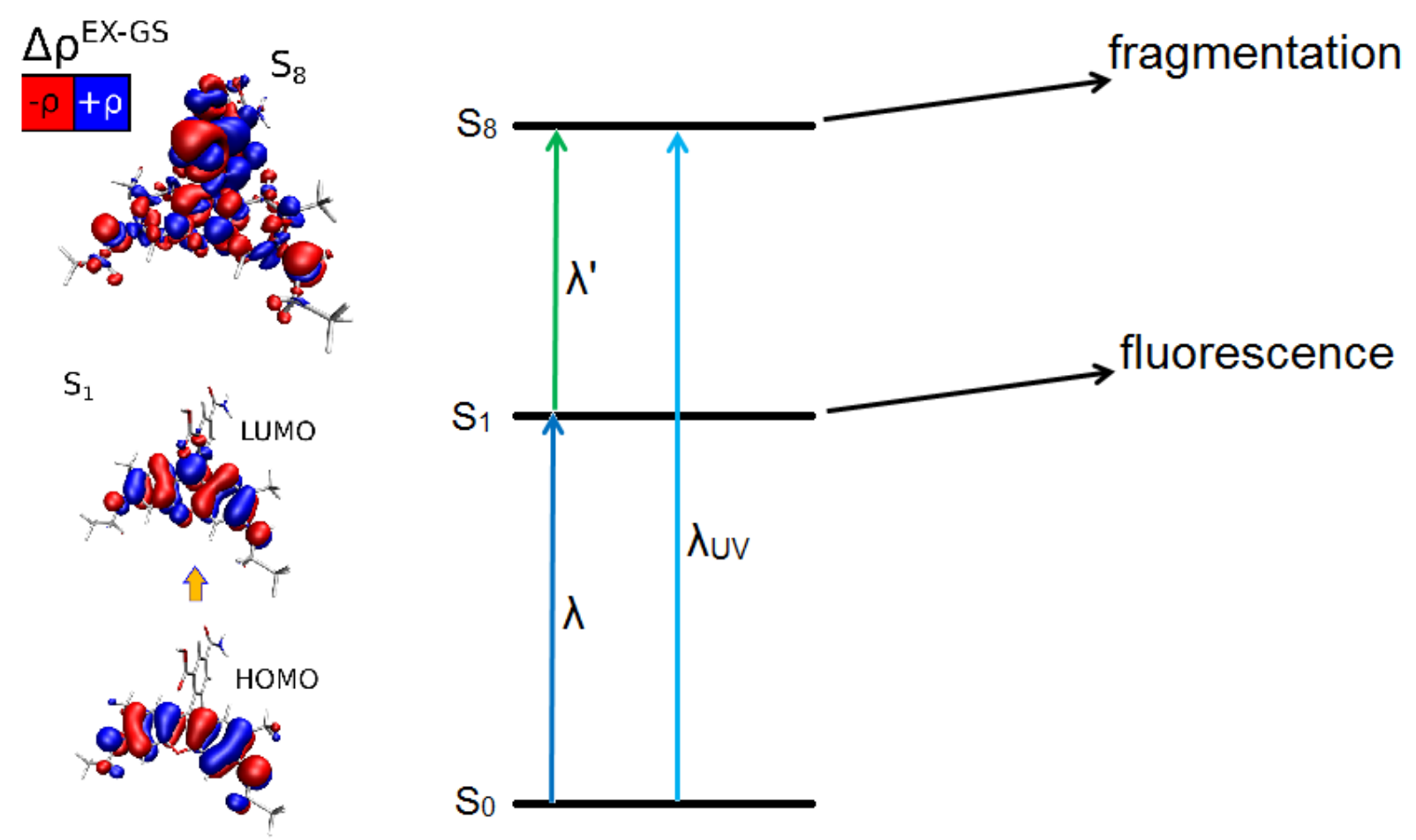
Visible and Ultraviolet Spectroscopy of Gas Phase Rhodamine 575 Cations.

Steven Daly ${ }^{\mathrm{a}, \mathrm{b}}$, Alexander Kulesza ${ }^{\mathrm{a}}$, Geoffrey Knight ${ }^{\mathrm{a}, \mathrm{b}}$, Luke MacAleese ${ }^{\mathrm{a}, \mathrm{b}}$, Rodolphe Antoine $^{\mathrm{a}, \mathrm{b}}$, Philippe Dugourd ${ }^{\mathrm{a}, \mathrm{b}}$.

${ }^{\text {a } U n i v e r s i t e ́ ~ d e ~ L y o n, ~ F-69622, ~ L y o n, ~ F r a n c e . ~}$

${ }^{\mathrm{b}}$ CNRS et Université Lyon 1, UMR5306, Institut Lumière Matière.

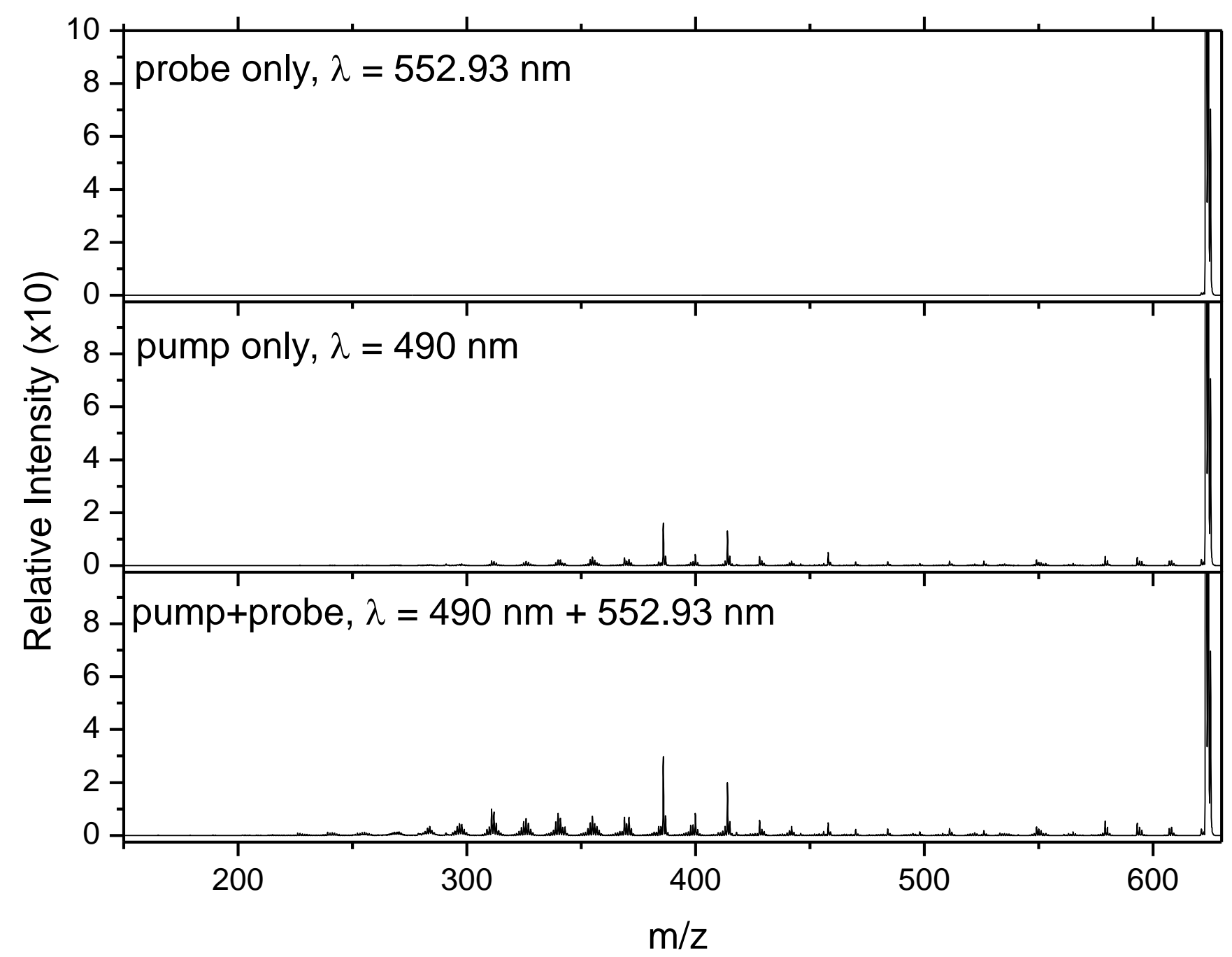

Figure S1. Mass spectra following irradiation with a single laser pulse of wavelength $552.93 \mathrm{~nm}$ (probe), $490 \mathrm{~nm}$ (pump) and both 552.93 and $490 \mathrm{~nm}$ simultaneously (pump + probe). 


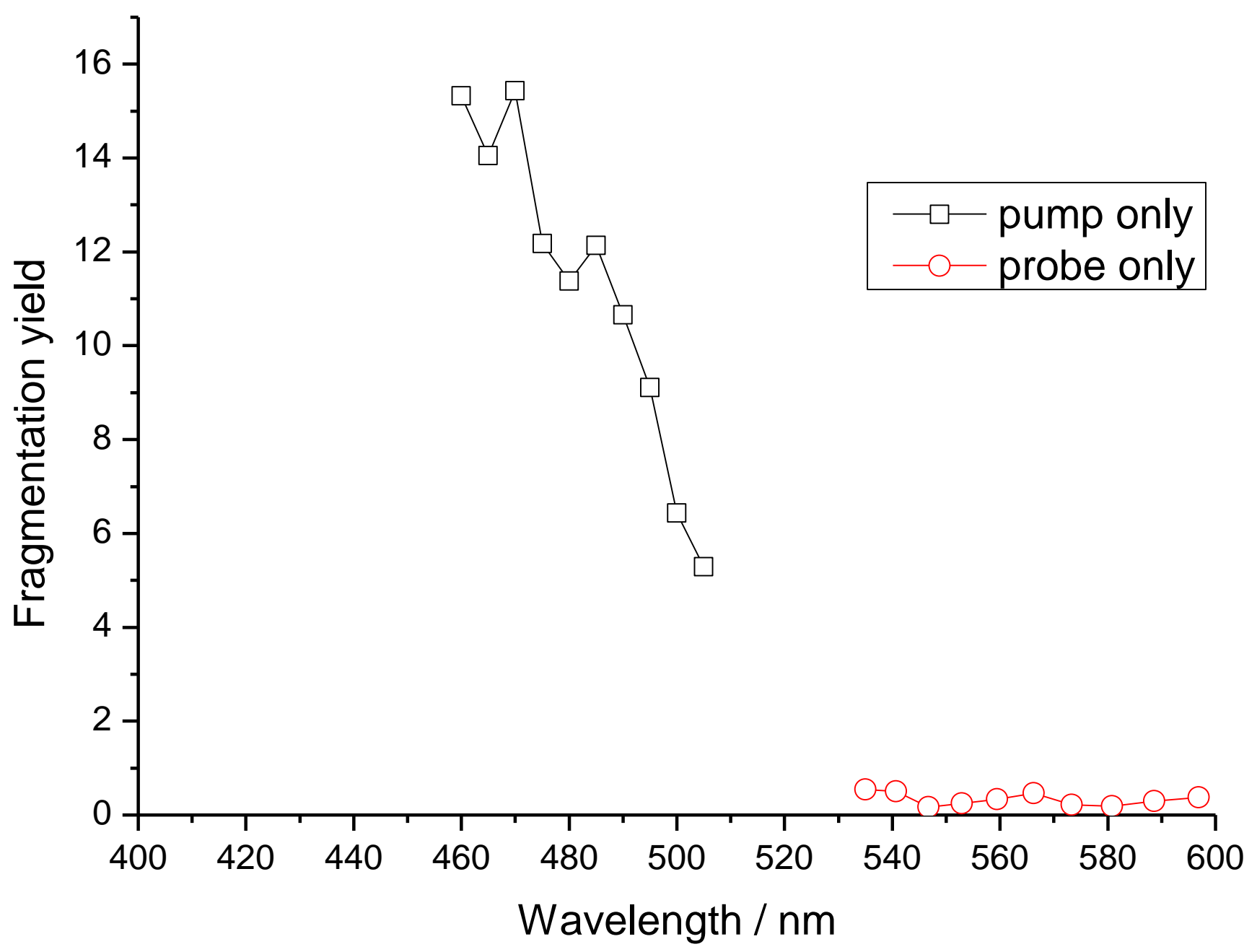

Figure S2. Fragmentation yield for pump only (black squares) and probe only (red circles)mass spectra measured concurrently with the 2-colour experiments. The probe laser wavelengths lie in a range where ground state R575 does not absorb, as evidenced by the flat profile for the fragmentation. 


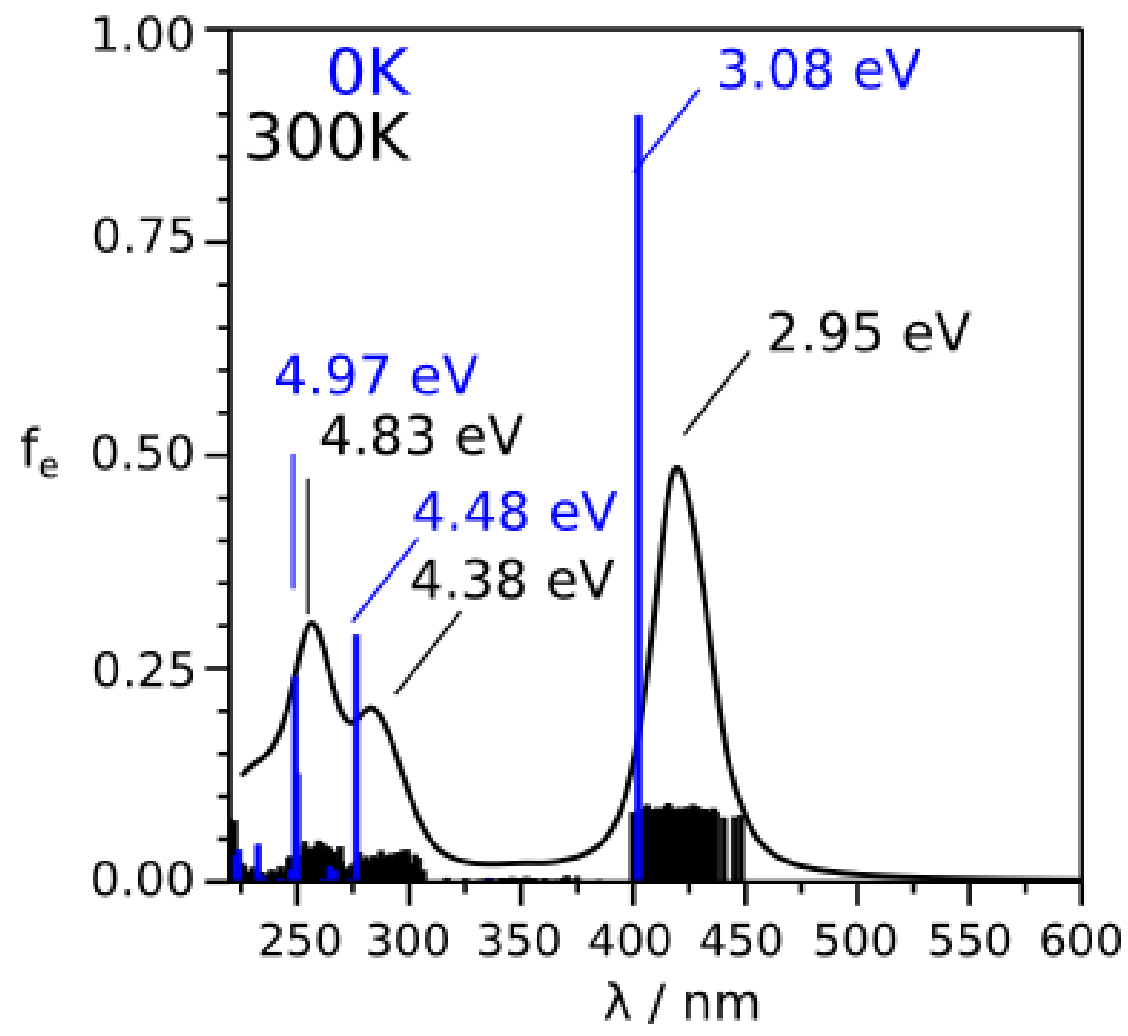

Figure S3 : Thermochromic shift from explicitly calculated TDDFT absorption spectra (oscillator strength $f_{e}$ versus wavelength $\lambda$ in $\mathrm{nm}$ ) upon a $300 \mathrm{~K}$ canonical ensemble obtained with semi-empirical MD. TDDFT calculations employ $\omega \mathrm{B} 97 \mathrm{XD} / 6-31+\mathrm{G}^{* *}$ level of theory (slightly smaller basis set does not significantly change transition energies and oscillator strengths, compare main text). Blue sticks indicate $0 \mathrm{~K}$ transitions, black line is the normalized convoluted (Lorentzian line shape $15 \mathrm{~nm}$ halfwidth) sum of 99 TDDFT spectra of the 300K structural ensemble (black sticks are the single transition energies and oscillator strengths scaled by a factor of 10 to better guide the eye). The $0 \mathrm{~K}$ transition energies and energies of the thermally broadened peak maxima are given also in $\mathrm{eV}$. 


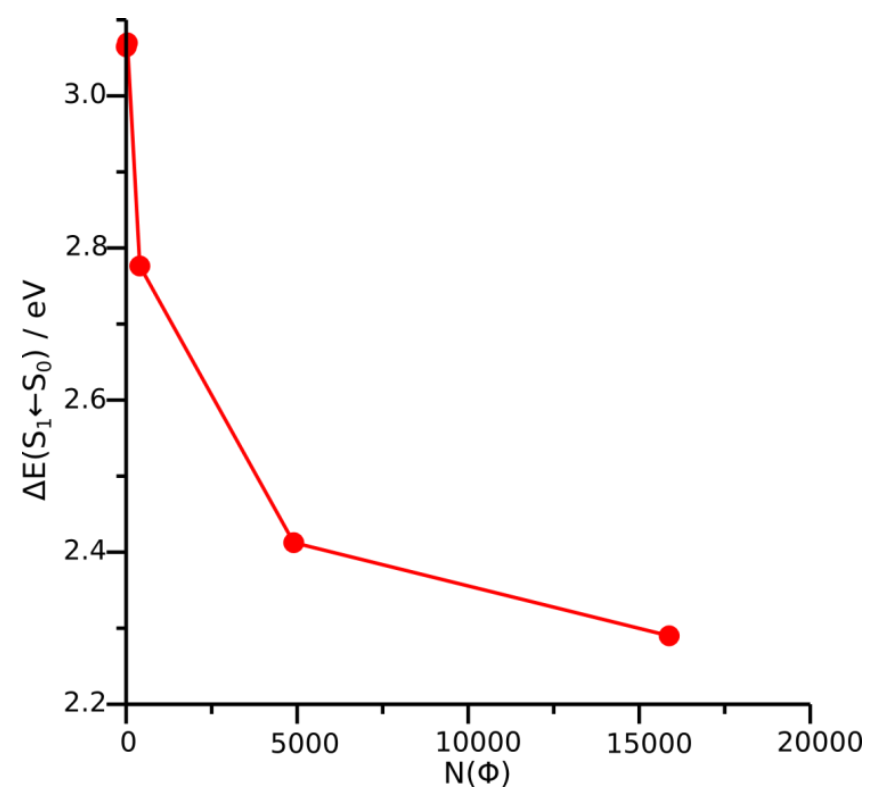

Figure $\mathrm{S} 4: \mathrm{S}_{1} \leftarrow \mathrm{S}_{0}$ transition energy $\Delta \mathrm{E}\left(\mathrm{S}_{1} \leftarrow \mathrm{S}_{0}\right)$ calculated with the semi-empirical multi-electron configuration interaction scheme based on the PM7 Hamiltonian (see table S1 for details) as a function of the number $\mathrm{N}$ of correlated configurations $\Phi$. 

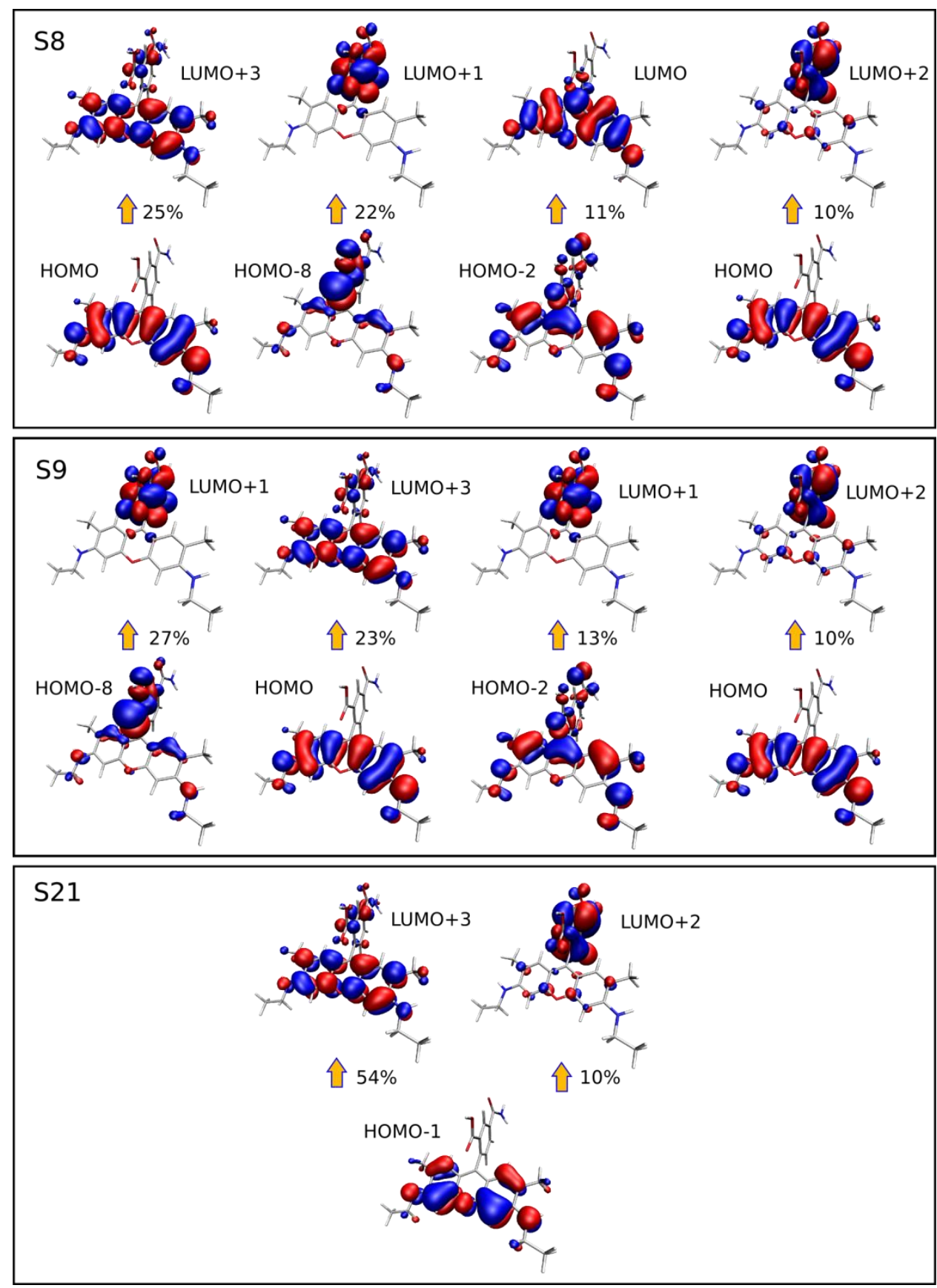

Figure S5 : Analysis of TDDFT calculations: Leading excitations of transition to the $S_{8}, S_{9}$ and $S_{21}$ electronic states (compare table S2) by visualization of involved Kohn-Sham orbitals (iso-surface value $=0.03$; red and blue parts indicate different sign of the orbital lobe) 
Table S1: Details of the semi-empirical multi-electron configuration interaction scheme based on the PM7 Hamiltonian. Active Space $(\mathrm{m}, \mathrm{n})$ with $\mathrm{m}$ electrons in $\mathrm{n}$ levels giving rise to $\mathrm{N}$ correlated configurations $\Phi$ and the calculated $\mathrm{S}_{1} \leftarrow \mathrm{S}_{0}$ transition energy $\Delta \mathrm{E}\left(\mathrm{S}_{1} \leftarrow \mathrm{S}_{0}\right)$.

\begin{tabular}{|l|l|l|}
\hline$(m, n)$ & $N(\Phi)$ & $\Delta E\left(S_{1} \leftarrow S_{0}\right) / e V$ \\
\hline 2,2 & 4 & 3.06 \\
\hline 4,4 & 36 & 3.06 \\
\hline 6,6 & 400 & 2.77 \\
\hline 8,8 & 4900 & 2.42 \\
\hline 8,9 & 15876 & 2.29 \\
\hline
\end{tabular}


Table S2: Details of TDDFT calculation for excited singlet electronic states $S_{1,3,8,9,21}$ : Transition energy from $\mathrm{S}_{0}$ electronic ground state (TE) / $\mathrm{eV}$, corresponding wavelength $\lambda / \mathrm{nm}$ as well as oscillator strength $\mathrm{f}_{\mathrm{e}}$. Leading excitations indicate Kohn-Sham orbitals 1-electron excitations contributing to more than $1 \%$.

\begin{tabular}{|c|c|c|c|c|}
\hline Excited State & TE /eV & $\lambda / \mathrm{nm}$ & $f_{e}$ & Leading excitations \\
\hline$S_{1}$ & 3.073 & 403.5 & 0.90 & HOMO $\rightarrow$ LUMO : $95 \%$ \\
\hline $\mathrm{S}_{3}$ & 4.475 & 277.0 & 0.25 & $\begin{array}{l}\text { HOMO-2 } \rightarrow \text { LUMO : } 65 \% \\
\text { HOMO-1 } \rightarrow \text { LUMO : } 10 \% \\
\text { HOMO-3 } \rightarrow \text { LUMO : } 9 \% \\
\text { HOMO }-8 \rightarrow \text { LUMO : } 3 \% \\
\text { HOMO } \rightarrow \text { LUMO+3 : } 2 \%\end{array}$ \\
\hline $\mathrm{S}_{8}$ & 4.935 & 251.3 & 0.19 & $\begin{array}{l}\text { HOMO } \rightarrow \text { LUMO+3 : } 25 \% \\
\text { HOMO-8 } \rightarrow \text { LUMO+1 : } 22 \% \\
\text { HOMO-2 } \rightarrow \text { LUMO+1 : } 11 \% \\
\text { HOMO } \rightarrow \text { LUMO+2 :10 \% } \\
\text { HOMO-4 } \rightarrow \text { LUMO+1 : } 4 \% \\
\text { HOMO-3 } \rightarrow \text { LUMO+1 : } 4 \% \\
\text { HOMO-8 } \rightarrow \text { LUMO+17 : } 2 \%\end{array}$ \\
\hline $\mathrm{S}_{9}$ & 4.946 & 250.7 & 0.17 & $\begin{array}{l}\text { HOMO-8 } \rightarrow \text { LUMO+1 : } 27 \% \\
\text { HOMO } \rightarrow \text { LUMO+3 : } 23 \% \\
\text { HOMO-2 } \rightarrow \text { LUMO+1 : } 13 \% \\
\text { HOMO } \rightarrow \text { LUMO+2 : } 10 \% \\
\text { HOMO-8 } \rightarrow \text { LUMO+17 : } 2 \% \\
\text { HOMO-2 } \rightarrow \text { LUMO : } 2 \%\end{array}$ \\
\hline$S_{21}$ & 5.907 & 209.8 & 1.30 & $\begin{array}{l}\text { HOMO-1 } \rightarrow \text { LUMO+3 : 54\% } \\
\text { HOMO-1 } \rightarrow \text { LUMO+2 : } 10 \% \\
\text { HOMO-9 } \rightarrow \text { LUMO : } 5 \% \\
\text { HOMO } \rightarrow \text { LUMO+6 : } 4 \% \\
\text { HOMO-2 } \rightarrow \text { LUMO+3 : } 3 \% \\
\text { HOMO } \rightarrow \text { LUMO+4 : } 3 \% \\
\text { HOMO } \rightarrow \text { LUMO+5 : } 3 \% \\
\text { HOMO-2 } \rightarrow \text { LUMO+2 } 3 \%\end{array}$ \\
\hline
\end{tabular}

